\title{
Avaliação da Reserva Ovariana: Comparação entre a Dosaģem do FSH Basal e 0 Teste do Clomifeno
}

\author{
Evaluation of Ovarian Reserve: Comparison Between Basal FSH Level and Clomiphene Test \\ Rodrigo Coelho Franco, Rui Alberto Ferriani, Marcos Dias Moura, Rosana Maria dos Reis, \\ Rodrigo Alves Ferreira, Maria Matheus de Sala
}

\section{RESUM0}

Objetivo: avaliar a reserva ovariana por meio da dosagem do FSH no $3^{\circ}$ dia do ciclo menstrual, comparando-o com o teste do clomifeno, e correlacionar os resultados com a resposta ovariana à hiperestimulação controlada com gonadotrofinas para a fertilização in vitro.

Métodos: foram selecionadas 49 pacientes com idade superior a 30 anos que apresentavam quadro clínico de infertilidade há pelo menos 1 ano. Foi realizada avaliação da reserva ovariana destas pacientes pelo teste do citrato de clomifeno. Posteriormente, 26 das 49 pacientes foram submetidas à hiperestimulação ovariana controlada com gonadotrofinas. Destas 26 pacientes, 18 tiveram boa resposta à hiperestimulação ovariana e 8, má resposta. Foram calculadas as médias e os desvios-padrão referentes aos valores do FSH do $3^{\circ}$ dia, do $10^{\circ}$ dia e do somatório de ambos, no grupo das pacientes que responderam favoravelmente à estimulação ovariana. Posteriormente foi realizada a correlação dos valores obtidos com a resposta ovariana após a estimulação dos ovários com gonadotrofinas.

Resultados: empregando-se a dosagem do FSH no $10^{\circ}$ dia (média somada a 2 desvios-padrão) com valor $\geq 16,1 \mathrm{UI} / \mathrm{mL}$ para predizer a má resposta ovariana ao teste do clomifeno, observaramse sensibilidade, especificidade e valores preditivos positivo e negativo de 50, 100, 100 e $81,8 \%$, respectivamente. Considerando-se o teste do clomifeno positivo quando o valor do somatório das dosagens do FSH do $3^{\circ}$ e do $10^{\circ}$ dia somado a dois desvios-padrão foi $\geq 22,6$ $\mathrm{UI} / \mathrm{mL}$, obtiveram-se sensibilidade de $62,5 \%$, especificidade de $100 \%$, valor preditivo positivo de $100 \%$ e valor preditivo negativo de $85,7 \%$. O uso da dosagem única do FSH no $3^{\circ}$ dia do ciclo acima de $10 \mathrm{UI} / \mathrm{mL}$ para predizer a má resposta ovariana mostrou sensibilidade de $87 \%$, especificidade de 100\%, valor preditivo positivo de 100\% e valor preditivo negativo de 94,7\%. Conclusão: no presente estudo, a dosagem única do FSH no $3^{\circ}$ dia do ciclo apresentou maior sensibilidade quando comparada ao teste do clomifeno para a avaliação da reserva ovariana.

PALAVRAS-CHAVE: Infertilidade. Reserva ovariana. Fertilização in vitro (FIV). Hiperestimulação ovariana. FSH.

\section{Introdução}

A quantidade de oócitos nos ovários humanos sofre rápida diminuição com o evoluir da ida-

Setor de Reprodução Humana do Departamento de Ginecologia e Obstetrícia do Hospital das Clínicas da Faculdade de Medicina de Ribeirão Preto da Universidade de São Paulo.

Correspondência:

Maria Matheus de Sala

Rua Rui Barbosa, 522 apto 112

14015-120 - Ribeirão Preto - SP de. Na vida intra-uterina, por volta da $20^{\mathrm{a}}$ semana de gestação, há aproximadamente 7.000.000 de oócitos, que ao nascimento estão reduzidos a cerca de 2.000.000. Na ocasião da menarca, esse número é de 200.000 a 300.000 oócitos, sendo que mensalmente há perda de grande número deles até a menopausa, quando não mais se encontra oócito algum ${ }^{1}$.

Nos casos de infertilidade, em que há a indicação da realização de procedimentos de técnicas de reprodução assistida, é prudente proceder à avaliação das reservas ovarianas antes da rea- 
lização de ciclos para fertilização in vitro (FIV) ou injeção intracitoplasmática de espermatozóides (ISCI), principalmente em pacientes com mais de 40 anos de idade. Nestes casos, a reserva ovariana pode estar naturalmente diminuída e o investimento em uma hiperestimulação ovariana pode não ser bem sucedido ${ }^{2}$.

Na tentativa de se avaliar a reserva ovariana, diversos testes foram propostos. Estes testes podem ser divididos em estáticos e dinâmicos ${ }^{3}$. Os testes estáticos são conhecidos como "testes basais" e comumente são realizados no terceiro dia do ciclo menstrual, destacando-se entre eles a dosagem basal do $\mathrm{FSH}^{4-6}$, a dosagem do $\mathrm{LH}^{7}$, a dosagem do estradiol ${ }^{8}$, a dosagem de inibina ${ }^{9-11}$, a medida do volume ovariano antes do GnRH-a ${ }^{12,13}$, e o número de pequenos folículos presentes antes da estimulação ${ }^{2,14}$.

Os testes dinâmicos avaliam a reserva pela mensuração dos hormônios FSH e/ou estradiol após uso de medicação. Entre estes testes, os mais empregados são o teste do clomifeno ${ }^{15-17}$, o teste da leuprolida ${ }^{18}$ e o teste do $\mathrm{FSH}^{19}$.

A dosagem do FSH no terceiro dia do ciclo é o método mundialmente mais utilizado para avaliar a reserva ovariana. Preenche os critérios de bom teste de triagem, pois é facilmente mensurável, minimamente invasivo, barato e apresenta bom valor preditivo ${ }^{6}$.

O teste do clomifeno, descrito por Navot et al. ${ }^{15}$, apesar de ser um método indireto de avaliação, é de fácil realização e parece ter boa sensibilidade e especificidade para a previsão da reserva ovariana.

Com base nas observações anteriores, o objetivo do presente estudo foi comparar dois métodos para avaliação da reserva ovariana: dosagem do FSH no terceiro dia do ciclo menstrual e o teste do clomifeno, e correlacionar os resultados com a resposta ovariana à hiperestimulação controlada com gonadotrofinas para a fertilização in vitro.

\section{Pacientes e Métodos}

Foram selecionadas 49 pacientes com idade superior a 30 anos, portadoras de dois ovários, atendidas no Ambulatório de Infertilidade do Hospital das Clínicas da Faculdade de Medicina de Ribeirão Preto da Universidade de São Paulo, que apresentavam quadro clínico de infertilidade há pelo menos um ano. Nenhuma das pacientes havia sido submetida anteriormente a procedimentos de reprodução assistida neste serviço.

As pacientes e seus parceiros, foram submetidos à investigação básica para o casal infértil (citologia cérvico-vaginal; colposcopia; dosagens hormonais de FSH, LH e PRL; sorologia para sífilis, hepatite B, HIV e rubéola; coleta de swab endocervical; ultra-sonografia transvaginal; histerossalpingografia; histeroscopia diagnóstica; laparoscopia diagnóstica; espermograma e espermocultura). Após estes exames, foi indicada a realização de fertilização in vitro em 26 pacientes, tendo-se excluído aquelas com indicação para a realização de inseminação intra-uterina e/ou as que apresentavam alterações que contra-indicassem o procedimento.

Foi realizada avaliação da reserva ovariana destas pacientes por meio do teste do citrato de clomifeno. Este teste consiste na dosagem do FSH basal no $3^{\circ}$ dia do ciclo menstrual, sem uso prévio de nenhuma medicação hormonal por um período de pelo menos três meses. Após a administração de citrato de clomifeno do $5^{\circ}$ ao $9^{\circ}$ dia do ciclo por via oral, na dosagem de $100 \mathrm{mg}$ ao dia, foi realizada nova dosagem de $\mathrm{FSH}$ no $10^{\circ}$ dia do ciclo (Figura 1).

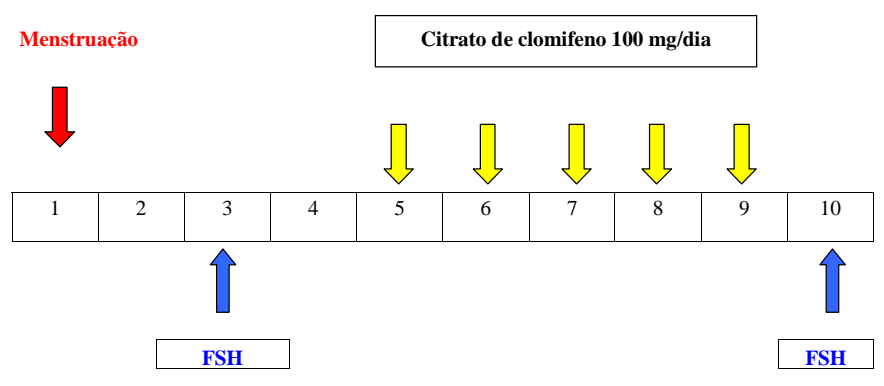

Figura 1 - Esquema do teste do citrato de clomifeno. Os números de 1 a 10 representam os dias do ciclo menstrual. Os níveis de FSH foram avaliados no $3^{\circ}$ e $10^{\circ}$ dia do ciclo. 0 citrato de clomifeno foi administrado do $5^{\circ}$ ao $9^{\circ}$ dia do ciclo (100 mg/dia).

Foram coletadas amostras de sangue de cada paciente no Laboratório de Reprodução Humana do HC-FMRP-USP, sempre no período da manhã. Estas amostras foram submetidas a centrifugação durante 10 minutos a $45.000 \mathrm{rpm}$ (Rotor IEC modelo 2K Boston, MA) e posteriormente procedeu-se à dosagem do FSH. As amostras foram dosadas em dias separados (diferentes kits do FSH) conforme o ciclo de cada paciente. Não houve congelamento de nenhuma amostra. O método utilizado para a dosagem do FSH foi o da quimioluminescência automatizada, realizada em um analisador Immulite (DPC Cirrus Inc., Los Angeles, CA, USA), cujo valor de referência para a normalidade varia de 2,6 a $10,0 \mathrm{UI} / \mathrm{mL}$.

Posteriormente, 26 das 49 pacientes foram submetidas à hiperestimulação ovariana controlada com gonadotrofinas, todas sob bloqueio hipofisário prévio com análogo do GnRh e estimu- 
lação com FSH, utilizando-se para este esquema o protocolo longo. Destas 26 pacientes, 18 tiveram boa resposta à hiperestimulação ovariana controlada. Considerou-se resposta satisfatória a produção de 4 ou mais folículos quando utilizado o FSH na dosagem de $300 \mathrm{UI} /$ dia durante pelo menos 5 dias.

Foram calculadas as médias e os desviospadrão referentes aos valores do $\mathrm{FSH}$ do $3^{\circ}$ dia e do $10^{\circ}$ dia e do somatório de ambos, no grupo de pacientes que responderam favoravelmente à estimulação ovariana. Posteriormente foi realizada a correlação dos valores obtidos com a resposta ovariana após a estimulação controlada dos ovários com gonadotrofinas.

A média de idade das pacientes que apresentaram uma boa resposta foi de 35,6 anos, com variação entre 31 e 40 anos.

Este estudo foi aprovado pela Comissão de Ética em Pesquisa do Hospital das Clínicas da Faculdade de Medicina de Ribeirão Preto da Universidade de São Paulo - HC-FMRP-USP. As pacientes e seus esposos assinaram termo de consentimento pós-informação. Neste sentido, os casais foram, antes do início do ciclo de tratamento, adequada e completamente informados sobre o procedimento da fertilização in vitro e transferência de embriões, os resultados e as leis que regulamentam a utilização da técnica.

\section{Resultados}

A dosagem do $\mathrm{FSH}$ do $3^{\circ}$ dia apresentou média de $5,5 \mathrm{UI} / \mathrm{mL}( \pm 2,01)$, enquanto que a dosagem média do $\mathrm{FSH}$ do $10^{\circ}$ dia foi de $9,1 \mathrm{UI} / \mathrm{mL} \pm^{ \pm}$ 3,5 ). A média do FSH total (somatório do FSH do $3^{\circ}$ dia e do FSH do $10^{\circ}$ dia) foi de $14,6( \pm 4,0) \mathrm{UI} / \mathrm{mL}$.

Considerando-se a dosagem do $\mathrm{FSH}$ do $10^{\circ}$ dia do ciclo $\geq 16,1 \mathrm{UI} / \mathrm{mL}$ (média +2 desvios padrão), como preconizado por Navot et al. ${ }^{15}$, para predizer a má resposta ovariana ao teste do clomifene, observaram-se sensibilidade, especificidade e valores preditivos positivo e negativo de 50, 100, 100 e $81,8 \%$, respectivamente (Figura 2).

Considerando-se o teste do clomifene como positivo para a má resposta ovariana quando o valor do somatório das dosagens do $\mathrm{FSH}$ do $3^{\circ}$ e do $10^{\circ}$ dia foi $\geq 22,6 \mathrm{UI} / \mathrm{mL}$, conforme recomendado por Loumaye et al. ${ }^{16}$, obtiveram-se sensibilidade de $62,5 \%$, especificidade de $100 \%$, valor preditivo positivo de $100 \%$ e valor preditivo negativo de $85,7 \%$ (Figura 2).

Considerando-se a dosagem única do $\mathrm{FSH}$ no $3^{\circ}$ dia do ciclo acima de $10 \mathrm{UI} / \mathrm{mL}$ para predizer a má resposta ovariana, obtiveram-se sensibili- dade de $87 \%$, especificidade de $100 \%$, valor preditivo positivo de $100 \%$ e valor preditivo negativo de $94,7 \%$ (Figura 2).

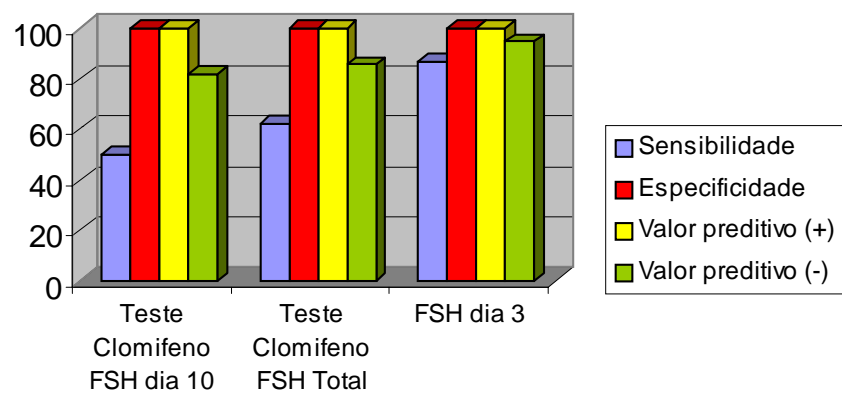

Figura 2 - Comparação entre os resultados obtidos na avaliação da reserva ovariana por meio da dosagem basal do FSH no $3^{\circ}$ dia do ciclo e do teste do clomifeno nas suas duas formas de interpretação (FSH no $10^{\circ}$ dia do ciclo e somatório das duas dosagens).

\section{Discussão}

Parâmetros como o FSH basal, inibina beta, estradiol, a resposta ovariana endócrina a um estímulo exógeno (teste do clomifeno, teste do análogo do GnRH, estimulação com FSH) e a medida do volume ovariano têm sido utilizados para predizer a resposta do ovário quando estimulado com gonadotrofinas ${ }^{20}$.

Os níveis de FSH na fase folicular precoce aumentam com o avançar da idade. Este aumento é atribuído a uma redução dos níveis da inibina ovariana pela diminuição da carga folicular. O mecanismo envolve sistema de retrocontrole do ovário sobre a hipófise, traduzindo indiretamente a carga oocitária ${ }^{21}$. A dificuldade na interpretação dos resultados está em adotar um valor de corte para o FSH a partir do qual se observa resposta ovariana diminuída. Vários estudos, nos quais se empregou a referida metodologia, relatam o valor prognóstico da dosagem do FSH basal para predizer a resposta ovariana. Neste sentido, nos casos em que a dosagem do FSH é elevada, a probabilidade do teste positivo para uma má resposta é grande, podendo-se aconselhar as pacientes a não realizar os ciclos de hiperestimulação ovariana ${ }^{21}$.

$\mathrm{O}$ aumento do FSH na fase folicular precoce é uma característica da idade reprodutiva avançada e tem se tornado método freqüentemente utilizado para determinar a reserva ovariana diminuída e para prever a má resposta. Entretanto, existem fatores que podem falsear a interpretação por elevarem os níveis de $\mathrm{FSH}^{22}$. Podem ser citados a amenorréia da lactação, anticoncepcionais orais, recuperação de perda de peso, amenorréia por estresse, intoxicação exógena (tabagismo) e causas iatrogênicas (ooforectomia uni- 
lateral) $^{22}$. Tomando-se os devidos cuidados na interpretação dos resultados, a dosagem do FSH basal é, no entanto, método simples e eficaz ${ }^{22}$.

O teste do clomifeno se baseia no mesmo raciocínio para a interpretação na avaliação da reserva ovariana. Entretanto, promove-se estimulação ovariana leve e indireta. Realizado no esquema descrito (Figura 1), os valores de FSH são avaliados antes e depois da administração do clomifeno. Nos casos de ovários com boa carga oocitária, a elevação do FSH do $10^{\circ}$ dia será suprimida por retrocontrole negativo exercido pelo estradiol e pela inibina produzidos pelos folículos em desenvolvimento. Caso não haja folículos, há elevação dos níveis FSH nesta segunda dosagem ${ }^{15}$.

$\mathrm{Na}$ interpretação do teste do clomifeno, alguns autores sugerem avaliar somente a segunda dosagem do FSH, considerando como valor limite $26 \mathrm{U} / 1^{15}$ ou a soma das dosagens do FSH, também com um valor próximo ${ }^{16}$. No entanto, a soma dos valores do FSH parece não mostrar uma melhor correlação com a idade em comparação ao uso da variável isoladamente ${ }^{21}$.

Independentemente do método utilizado, no grupo de pacientes estudadas, os resultados demonstraram que a sensibilidade da dosagem isolada do FSH no $3^{\circ}$ dia do ciclo menstrual foi maior que a sensibilidade do teste do clomifeno efetuado segundo os esquemas utilizados por Navot et al. ${ }^{15}$ e Loumaye et al. ${ }^{16}$.

Importante observação está relacionada com os métodos utilizados para a dosagem do FSH. Os estudos de Navot et al. ${ }^{15}$ e Loumaye et al. ${ }^{16}$ usaram como método de dosagem o radioimunoensaio e no presente trabalho utilizou-se a quimioluminescência, cujos valores de referência são diferentes. Outra observação se refere à existência de diversos kits comerciais de dosagens hormonais no mercado, havendo, portanto, a necessidade de cada serviço determinar seus próprios valores de referência.

\section{ABSTRACT}

Purpose: to assess ovarian reserve by FSH determination on the $3 r d$ day of the menstrual cycle compared to the clomiphene test and to correlate the results with the ovarian response to controlled hyperstimulation with gonadotrophins for in vitro fertilization.

Methods: a total of 49 patients older than 30 years who had been presenting a clinical picture of infertility for at least 1 year were selected. All patients were evaluated for ovarian reserve by the clomiphene citrate test and 26 of them were later submitted to controlled ovarian hyperstimulation with gonadotrophins. Of these 26 patients, 18 showed a good response to ovarian hyperstimulation and 8 showed a poor response. Mean ( $\pm S D)$ FSH values were calculated for the determinations on the $3^{\text {rd }}$ and on the $10^{\text {th }}$ day and for their sum in the group of patients who responded favorably to ovarian stimulation, and were later correlated with the ovarian response after gonadotrophin stimulation.

Results: employing a FSH value $\geq 16.1 \mathrm{IU} / \mathrm{mL}$ on the $10^{\text {th }}$ day (mean plus 2 SD) for the prediction of a poor ovarian response in the clomiphene test, the sensitivity, specificity, and positive and negative predictive values of this parameter were 50 , 100, 100 and $81.8 \%$, respectively. Considering the clomiphene test to be positive when the sum of the FSH values determined on the $3^{r d}$ and $10^{\text {th }}$ day plus $2 S D$ was $\geq 22.6$ IU/ $m L$, we obtained $62.5 \%$ sensitivity $100 \%$ specificity, $100 \%$ positive predictive value, and $85.7 \%$ negative predictive value. A single FSH determination of $10 \mathrm{IU} / \mathrm{mL}$ on the $3^{\text {rd }}$ day of the cycle for the prediction of a poor ovarian response showed $87 \%$ sensitivity, $100 \%$ specificity, $100 \%$ positive predictive value and $94.7 \%$ negative predictive value.

Conclusion: in the present study, a single FSH determination on the $3^{\text {rd }}$ day of the cycle showed to be more sensitive than the clomiphene test for the evaluation of ovarian reserve.

KEY WORDS: Infertility. Ovarian reserve. In vitro fertilization $(I V F)$. Ovarian hyperstimulation. FSH.

\section{Referências}

1. Speroff L, Glass RH, Kase NG. O ovário desde a concepção até a senilidade. In: Speroff L, Glass RH, Kase NG, editors. Endocrinologia Ginecológica, Clínica e Infertilidade. $4^{a}$ ed. São Paulo: Manole; 1991. p.103-43.

2. Tomas C, Nuojua-Huttunen S, Martikainen H. Pretreatment transvaginal ultrasound examination predicts ovarian responsiveness to gonadotrophins in in-vitro fertilization. Hum Reprod 1997; 12:220-3.

3. Younis JS, Haddad S, Matilsky M, Ben-Ami M. Premature luteinization: could it be an early manifestation of low ovarian reserve? Fertil Steril 1998; 69: 461-5.

4. Cameron IT, O'Shea FC, Rolland JM, Hughes EG, de Kretser DM, Healy DL. Occult ovarian failure: a syndrome of infertility, regular menses, and elevated follicle-stimulating hormone concentrations. J Clin Endocrinol Metab 1988; 67:1190-4.

5. Scott RT Jr. Evaluation and treatment of low responders. Semin Reprod Endocrinol 1996; 14:317-37.

6. Gürgan T, Urman B, Yarali H, Duran HE. Folliclestimulating hormone levels on cycle day 3 to predict ovarian response in women undergoing controlled ovarian hyperstimulation for in vitro fertilization using a flare-up protocol. Fertil Steril 1997; 68:483-7. 
7. Noci I, Biagiotti R, Maggi M, Ricci F, Cinotti A, Scarselli G. Low day 3 luteinizing hormone values are predictive of reduced response to ovarian stimulation. Hum Reprod 1998; 13:531-4.

8. Evers JL, Slaats P, Land JA, Dumoulin JC, Dunselman GA. Elevated levels of basal estradiol17 beta predict poor response in patients with normal basal levels of follicle stimulating hormone undergoing in vitro fertilization. Fertil Steril 1998; 69:1010-4.

9. Seifer DB, Lambert-Messerlian G, Hogan JW, Gardiner AC, Blazar AS, Berk CA. Day 3 serum inhibin-B is predictive of assisted reproductive technologies outcome. Fertil Steril 1997; 67:110-4.

10.Danforth DR, Arbogast LK, Mroueh J, et al. Dimeric inhibin: a direct marker of aging. Fertil Steril 1998; 70:119-23.

11.Hofmann GE, Danforth DR, Seifer DB. Inibin-B: the physiologic basis of the clomiphene citrate challenge test for ovarian reserve screening. Fertil Steril 1998; 69:474-7.

12.Syrop CH, Willhoite A, Van Voorhis BJ. Ovarian volume: a novel outcome predictor for assisted reproduction. Fertil Steril 1995; 64:1167-71.

13.Bazeos A, Tozer A, Zosmer A, Al-Shawaf T, Lower A, Grudzinskas JG. Pre-treatment transvaginal ultrasound measurement of the ovarian volume: a predictor of responsiveness in an in-vitro fertilization cycle. Hum Reprod 1998; 13:26-7.

14. Khalifa EE. Transvaginal ultrasound ovarian morphology: can it predict poor ovarian response to gonadotrophin stimulation in IVF? Hum Reprod 1998; 13:39-40.
15.Navot D, Rosenwaks Z, Margalioth EJ. Prognostic assessment of female fecundity. Lancet 1987; 2:645-7.

16.Loumaye E, Billion JM, Mine JM, Psalti I, Pensis M, Thomas K. Prediction of individual response to controlled ovarian hyperstimulation by means of a clomiphene citrate challenge test. Fertil Steril 1990; 53:295-301.

17.Scott RT, Leonardi MR, Hofmann GE, Illions EH, Neal GS, Navot D. A prospective evaluation of clomiphene citrate challenge test screening of the general infertility population. Obstet Gynecol 1993; 82:539-44.

18.Padilla SL, Dugan K, Maruschak V, Shalika S, Smith $\mathrm{RD}$. Use of the flare-up protocol with high dose human follicle stimulating hormone and human menopausal gonadotropins for in vitro fertilization in poor responders. Fertil Steril 1996; 65:796-9.

19.Fanchin R, de Ziegler D, Olivennes F, Taieb J, Dzik A, Frydman R. Exogenous follicle-stimulating hormone ovarian reserve test (EFORT): a simple and reliable screening test for detecting poor responders in in-vitro fertilization. Hum Reprod 1994; 9:1607-11.

20.Karande V, Gleicher N. A rational approach to the management of low responders in in-vitro fertilization. Hum Reprod 1999; 14:1744-8.

21.Broekmans FJ, Scheffer GJ, Bancsi LF, Dorland M, Blankenstein MA, te Velde ER. Ovarian reserve tests in infertility practice and normal fertile women. Maturitas 1998; 30:205-14.

22.Lambalk $\mathrm{CB}$, de Koning $\mathrm{CH}$. Interpretation of elevated FSH in the regular menstrual cycle. Maturitas 1998; 30:215-20.

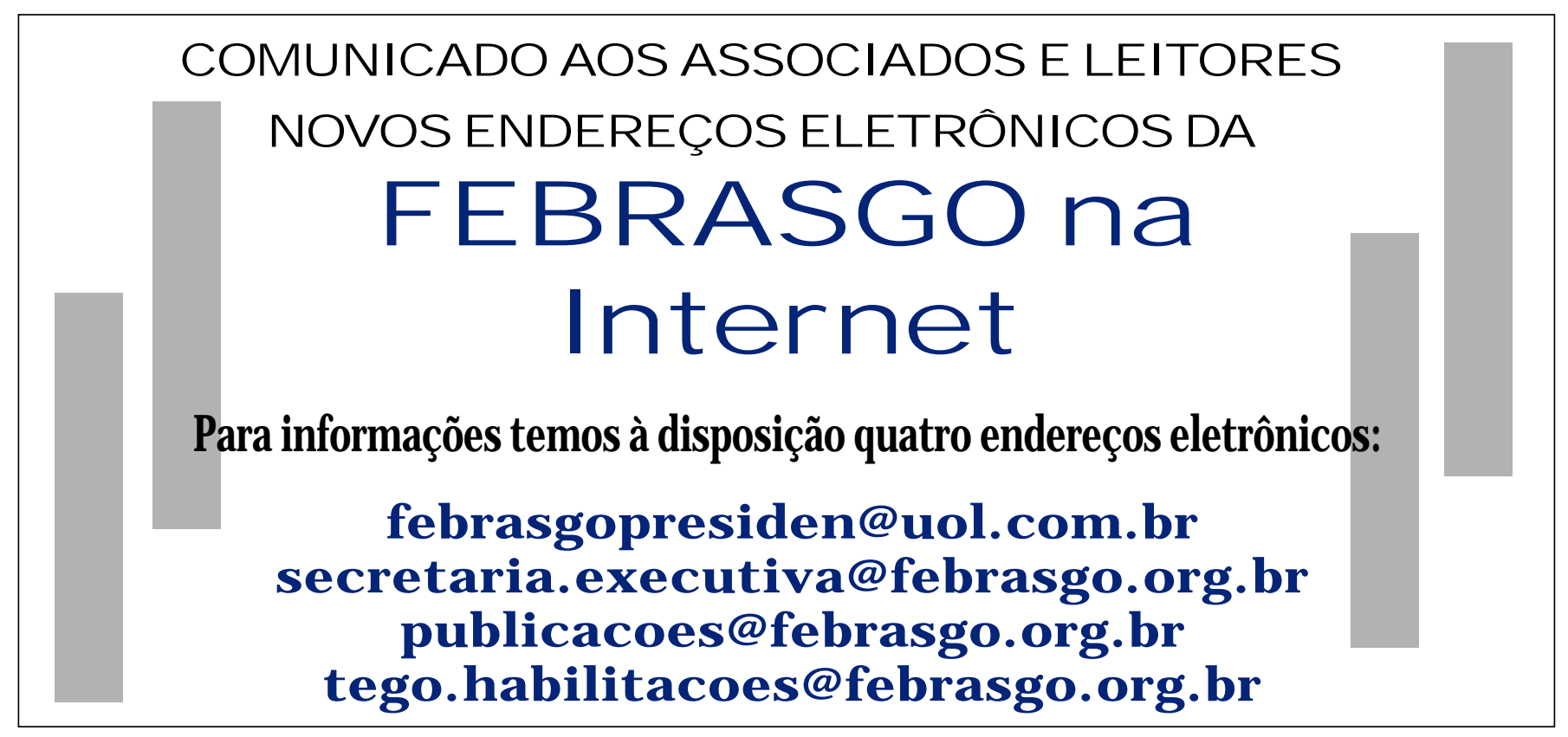

